\title{
Abeta Induces Abnormal Cytoskeletal Dynamics which are Reversible Upon Peptide Removal
}

\author{
A. G. Henriques, S. I. Vieira and O. A. B. da Cruz e Silva \\ Neuroscience Lab, Center for Cell Biology, Health Sciences Dept. and Biology Dept, University of \\ Aveiro, Campus de Santiago, 3810-193 Aveiro, Portugal
}

\begin{abstract}
Alzheimer's disease (AD) is characterized by extensive neuronal loss in brain areas related to memory and cognitive functions. Central to the neurodegenerative process is a peptide termed Abeta. The latter is the main component of senile plaques, one of the histopathological hallmarks of $\mathrm{AD}$, and derives from proteolytic processing of the Alzheimer's amyloid precursor protein (APP). Among the alterations induced by Abeta is increased cellular oxidative stress, imbalanced protein phosphorylation and cytoskeletal abnormalities, all factors that contribute to neuronal death.
\end{abstract}

Previous studies from our laboratory have shown that Abeta leads to intracellular accumulation of the secreted form of APP (sAPP). Consequently we addressed how Abeta may affect cellular morphology or intracellular structures and lead to this phenomenon. Interestingly, alterations in the cytoskeletal network were clearly evident $[1,2]$. Namely, sAPP was seen to cluster in cytoskeletal-associated vesicular-like structures, which accumulate close to the plasma membrane [2].

Furthermore, and as revealed by microscopy analyses, Abeta incubation for $24 \mathrm{hr}$ also led to rearrangements in the cytoskeletal network in HeLa cells. The re-organization (Figure 1, Abeta) is striking as detected by the phalloidin staining, with F-actin polymerization being markedly increased. Cytoskeletal abnormalities compromise neuronal transport of neurotransmitters and other substances, important to neuronal survival and signalling. Thus, Abeta induced cytoskeletal anomalies are consistent with the cognitive deficits associated with Alzheimer's disease. Of note, Abeta removal in the last $3 \mathrm{hr}$ of the incubation period (Figure 1, Abeta-Abeta) was able to reverse the effects. These novel findings are significant as they show for the first time that the Abeta-induced effects on cytoskeletal reorganization can be reversed by the simple removal of the toxic Abeta peptide. The mode of action needs to be closely addressed, however, it is relevant to consider that Abeta has been shown to inhibit phosphoprotein phosphate 1 (PPP1) activity and the latter is implicated in cytoskeletal dynamics [3]. Further, as cytoskeleton impairment may mediate part of Abeta toxic effects, drugs targeting cytoskeletal network may also be of therapeutic value in Alzheimer's disease.

Acknowledgements: This work was supported by the Fundação para a Ciência e Tecnologia of the Portuguese Ministry of Science and Technology, the European Union (cNeupro), Center for Cell Biology UA, REEQ/1023/BIO/2005, PTDC/QUI-BIQ/101317/2008, POCTI/BIA-BCM/58469/2004, and the scholarship awarded to AGH - SFRH/BPD/45611/2008.

\section{References}

1. Henriques et al., J. Neurochem. 113:761-71, 2010

2. Henriques et al., J. Neurosci. Res. 87:1449-61, 2009

3. Vintém et al., Neurotoxicol. Teratol. 31:85-88, 2009 
DAPI F-actin Overlay

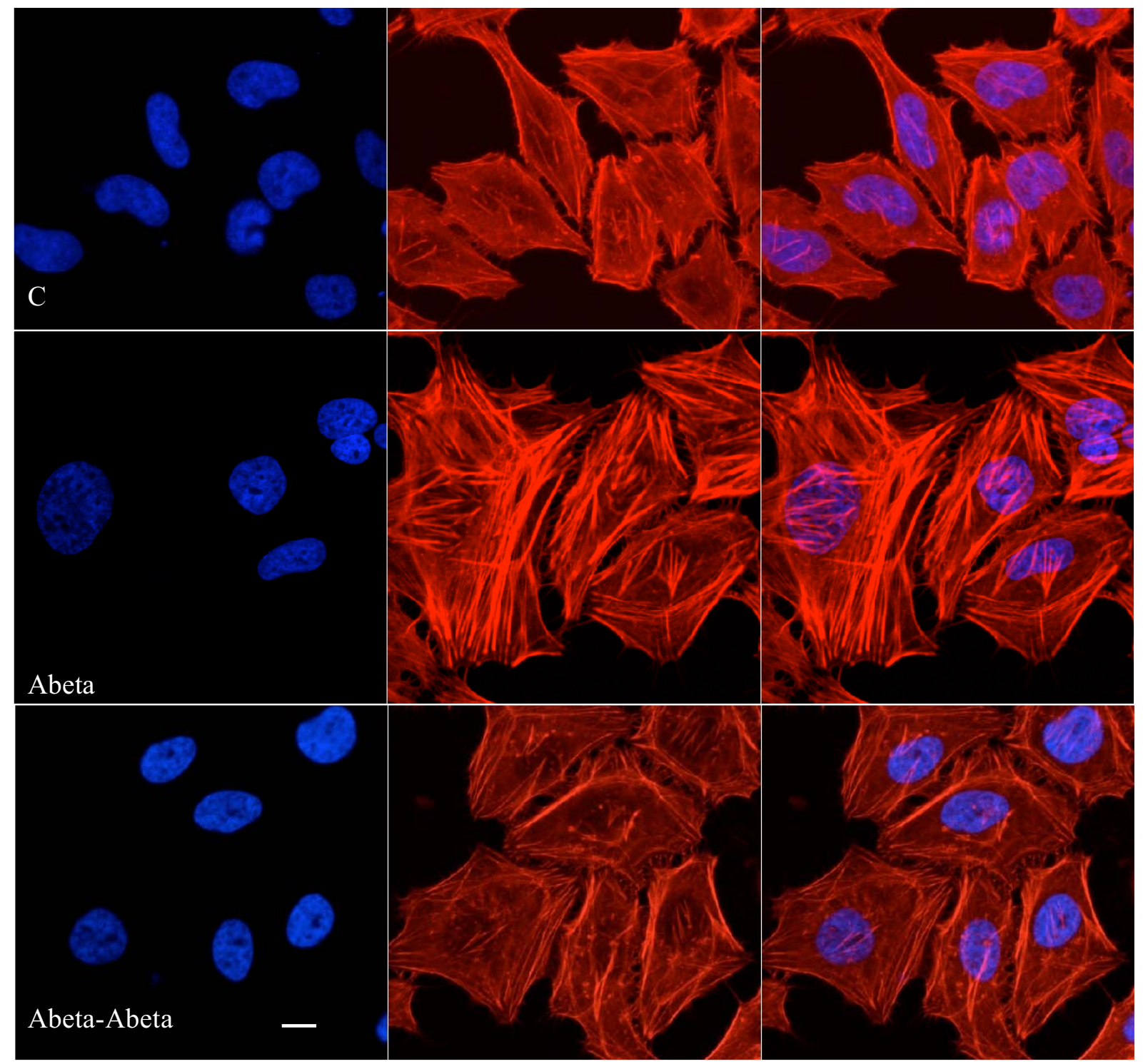

Figure 1. Abeta induced cytoskeletal reorganization is reversible. HeLa cells were treated with Abeta for $24 \mathrm{hr}$ (panel $\mathrm{C}$ untreated) and in the panel Abeta-Abeta, the peptide was removed for the last $3 \mathrm{hr}$ of the incubation period. HeLa cells were stained with Alexa Fluor 568 conjugated phallotoxin solution (labels filamentous F-actin). Blue- DAPI nuclear staining. Epifluorescent images were acquired using a LSM 510-Meta confocal microscope. Scale bar, $10 \mu \mathrm{m}$. 